\title{
An Example on Maximum Likelihood Estimates
}

LEONARD W. DEATON

Naval Postgraduate School

Monterey, California

In most introductory courses in mathematical statistics, students see examples and work problems in which the maximum likelihood estimate (MLE) of a parameter turns out to be either the sample mean, the sample variance, or the largest, or the smallest sample item. The purpose of this note is to provide an example in which the MLE is the sample median and a simple proof of this fact.

Suppose a random sample of size $n$ is taken from a population with the Laplace distribution $f(x ; \theta)=$ $\left(\frac{1}{2}\right) \exp (-|x-\theta|)$. The mean, mode, and median of this distribution is $\theta$. The sample median is the MLE of $\theta$ ([1], page 247).

Proof: The likelihood function is

$$
L(\theta)=\left(\frac{1}{2}\right)^{n} \exp \left(-\sum_{i=1}^{n}\left|x_{i}-\theta\right|\right) .
$$

Now, $L$ is maximum when

$$
\sum_{i=1}^{n}\left|x_{i}-\theta\right|
$$

is minimum. It will be shown that the inequality,

$$
\sum_{i=1}^{n}\left|x_{i}-\theta\right| \geq \sum_{i=1}^{n}\left|x_{i}-m\right|
$$

where $m$ is the sample median, holds for every value of $\theta$. Consider two cases.

Case I: Let $n$ be even. Let $y_{1}, y_{2}, y_{3}, \cdots, y_{n}$ be the observed values of the order statistics. Let $m$ be any real number between the two middle values so that we have

$$
y_{1} \leq y_{2} \leq \cdots y_{n / 2} \leq m \leq y_{(n / 2)+1} \leq \cdots \leq y_{n} .
$$

In particular, $m$ may be the sample median. By application of (1) and the triangle inequality we see that

$$
\begin{aligned}
& \left|y_{i}-m\right|+\left|m-y_{n+1-i}\right| \\
& \quad=\left|y_{i}-y_{n+1-i}\right| \leq\left|y_{i}-\theta\right|+\left|\theta-y_{n+1-i}\right|
\end{aligned}
$$

for $i=1,2, \cdots, n / 2$ and $\theta$ any real number.

Upon summing in (2) we get

$$
\begin{aligned}
\sum_{i=1}^{n / 2}\left(\left|y_{i}-m\right|\right. & \left.+\left|m-y_{n+1-i}\right|\right) \\
& \leq \sum_{i=1}^{n / 2}\left(\left|y_{i}-\theta\right|+\left|\theta-y_{n+1-i}\right|\right) .
\end{aligned}
$$

Now, since

$$
\sum_{i=1}^{n / 2}\left|m-y_{n+1-i}\right|=\sum_{i=(n / 2)+1}^{n}\left|m-y_{i}\right|
$$

and

$$
\sum_{i=1}^{n / 2}\left|\theta-y_{n+1-i}\right|=\sum_{i=(n / 2)+1}^{n}\left|\theta-y_{i}\right|,
$$

(3) becomes

$$
\sum_{i=1}^{n}\left|y_{i}-m\right| \leq \sum_{i=1}^{n}\left|y_{i}-\theta\right| .
$$

But,

$$
\sum_{i=1}^{n}\left|y_{i}-m\right|=\sum_{i=1}^{n}\left|x_{i}-m\right|
$$

and

$$
\sum_{i=1}^{n}\left|y_{i}-\theta\right|=\sum_{i=1}^{n}\left|x_{i}-\theta\right| .
$$

The desired results follows.

Case II: Let $n$ be odd. Then $y_{(n+1) / 2}$ is the sample median. As in (2) above, we have

$$
\begin{aligned}
\mid y_{i} & -y_{(n+1) / 2}|+| y_{(n+1) / 2}-y_{n+1-i} \mid \\
& =\left|y_{i}-y_{n+1-i}\right| \leq\left|y_{i}-\theta\right|+\left|\theta-y_{n+1-i}\right|
\end{aligned}
$$

for $i=1,2, \cdots,(n+1) / 2-1$, and $\theta$ any real number. Also,

$$
0=\left|y_{(n+1) / 2}-y_{(n+1) / 2}\right| \leq\left|y_{(n+1) / 2}-\theta\right|
$$

By summing in (5), we see that

$$
\begin{aligned}
& \sum_{i=1}^{(n+1) / 2-1}\left(\left|y_{i}-y_{(n+1) / 2}\right|+\left|y_{(n+1) / 2}-y_{n+1-i}\right|\right) \\
& \leq \sum_{i=1}^{(n+1 / 2)-1}\left(\left|y_{i}-\theta\right|+\left|\theta-y_{n+1-i}\right|\right)
\end{aligned}
$$

But,

$$
\begin{aligned}
\sum_{i=1}^{(n+1) / 2-1}\left|y_{(n+1) / 2}-y_{n+1-i}\right| & \\
= & \sum_{i=(n+1) / 2+1}^{n}\left|y_{(n+1) / 2}-y_{i}\right|
\end{aligned}
$$

and

$$
\sum_{i=1}^{(n+1) / 2-1}\left|\theta-y_{n+1-i}\right|=\sum_{i=(n+1) / 2+1}^{n}\left|y_{i}-\cdot \theta\right| .
$$

Hence, using (6) and (8) in (7), we have

$$
\sum_{i=1}^{n}\left|y_{i}-y_{(n+1) / 2}\right| \leq \sum_{i=1}^{n}\left|y_{i}-\theta\right| .
$$

Now, after putting $y_{(n+1) / 2}=m$ and using (4), the proof is complete.

\section{RFFERENCE}

1. Hogg and Craig, Introduction to Mathematical Statistics, Macmillan, (1965). 